\title{
O uso de mobile learning no ensino de algoritmos
}

\author{
Ricardo JS Barcelos, Instituto Federal Fluminense, ricardo@iff.edu.br \\ Liane Tarouco, UFRGS, liane@ penta.ufrgs.br \\ Magda Berch, UFRGS, bercht@inf.ufrgs.br
}

Resumo. O estudo de algoritmos é uma área fundamental na computação que constitui uma das disciplinas com maior índice de reprovação. Este artigo analisa as principais causas das dificuldades enfrentadas pelo estudante no aprendizado de algoritmos usando como bases referenciais teóricos relacionados com aprendizagem significativa. A seguir investiga soluções existentes em termos de utilização de TICs para apoiar o processo de ensino aprendizagem de algoritmos com ênfase no uso de dispositivos móveis.

Abstract. The study of algorithms it is a basic area in computer science that constitutes one of the courses with bigger student failures rate. This paper analyzes the main causes of the difficulties faced by students using learning theories related to significant learning and applied to algorithms instruction. The article investigates existing solutions in terms of using computer technology to support learning process learning related to algorithms with emphasis in the use of mobile devices.

Palavras chaves: algoritmos, dispositivos móveis, educação móvel

\section{Introdução}

O estudo de algoritmos é uma área fundamental no campo da Computação - Engenharia da Computação, Bacharelado em Sistemas de Informação, entre outros - e das Engenharias não-computacionais como Engenharia de Controle e Automação, Engenharia Elétrica, entre outras, o conhecimento de algoritmos precede a aprendizagem de linguagens de programação, pois estes contem a essência da estratégia de solução.

Todavia, a aprendizagem de algoritmos constitui um dos grandes problemas enfrentados pelos alunos que ingressam nestes cursos e é a disciplina que aborda tais conhecimentos uma das que tem maior índice de reprovação. Um levantamento realizado pelos autores no IFF-Campos-RJ, aponta resultados apresentados na figura 1.

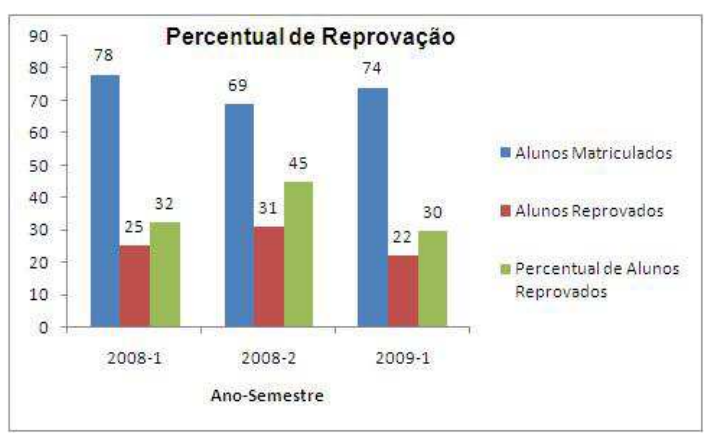

Figura 1: Índice de aprovação e reprovação em Algoritmos 
As causas para este alto nível de insucesso não são específicas da área, pois de modo geral muitos autores apontam que os alunos não apresentam desenvoltura na organização de raciocínios, elaboração de estratégias de resolução de problemas, atenção, concentração, estímulo ao processo de cálculo mental. Sendo assim, as habilidades envolvidas nesse processo, tais como, tentar, observar, conjeturar, deduzir e que compõem o que chamamos de raciocínio lógico, não estando apropriadamente desenvolvidas, interferem na aprendizagem de praticamente todas as áreas do conhecimento, mas em especial afetam esta área do conhecimento.

Em contrapartida, muitos destes alunos demonstram uma desenvoltura ímpar na utilização de recursos tecnológicos. Para dirimir esta dificuldade, aproveitando a motivação e vocação dos alunos para o uso da tecnologia, novas estratégias vêm sendo estudadas no que diz respeito ao uso de recursos de informática na educação, com a finalidade de potencializar habilidades que visam o desenvolvimento do raciocínio, Grabe (2001).

Neste artigo serão apresentados resultados da investigação do uso dos recursos atualmente disponíveis na computação móvel com vistas a identificar possibilidade de uso para atender as demandas da área de ensino aprendizagem de algoritmos. Este trabalho discute alguns resultados atuais no âmbito de pesquisas voltadas ao delineamento de uma arquitetura pedagógica que contemple ações que visem correlacionar estilos cognitivos e estilos de aprendizagem na área de algoritmos, com as características funcionais de dispositivos portáteis, usados num contexto de suporte à aprendizagem com mobilidade.

O ensino de algoritmos nas instituições tem sido constantemente, objeto de estudos, visando a melhoria do desempenho dos estudantes, pois este conteúdo é considerado, pelos professores, de suma importância sua trajetória acadêmica. A aprendizagem de algoritmos tem se constituído numa tarefa de complexidade elevada na quais podem destacar, conforme Pimentel e Omar (2008) a necessidade de desenvolver e/ou aprender habilidade para resolução de problemas, sintaxe e a semântica de uma linguagem de programação bem como a utilização de um ambiente de programação e realização de testes e depuração de programas. Estes autores destacam ainda que a aprendizagem de algoritmos tenha sido problemática em função de alguns fatores inerentes a este contexto específico tais como o raciocínio lógico que é um ponto crítico devido às dificuldades de compreensão que os alunos evidenciam bem como as dificuldades de representação de suas abstrações.

Jenkins (2002) aponta outras causas para o fraco desempenho nas disciplinas que envolvem algoritmos e programação: i) falta de competências para resolução de problemas; ii) inadequação dos métodos pedagógicos ao estilo de aprendizagem dos alunos, iii) linguagens de programação que possuem sintaxes adequadas para profissionais mas não para estudantes em inicio de curso.

As estratégias de ensino têm buscado criar condições para que o aluno exercite o pensamento lógico de forma análoga à maneira como a lógica é utilizada pelo computador, isto é, realizar operações lógicas de forma idêntica ao computador. As tarefas típicas buscar ensejar o desenvolvimento de habilidades que permitam resolver problemas relacionados à lógica de programação. Isto significa dizer que a capacidade 
do aluno, através da resolução de um problema, construir uma solução de forma ordenada, ainda que de forma não computacional não se encontra perfeitamente equacionada.

Os autores na prática docente destacam a dificuldade no ensino de algoritmos, derivada da necessidade de entender o processo de construção do algoritmo e propiciar a transferência efetiva de conhecimentos e habilidades educacionais, o que constitui desafio para o processo ensino aprendizagem, conforme afirma Santos (2008). Colocar o estudo de algoritmo no inicio do curso tem por objetivo desenvolver a habilidade de criar estratégias para organizar as idéias, o raciocínio, a abstração e a representação simbólica a fim de que o aluno incorpore estes hábitos e utilize-os sempre na resolução de problemas durante o próprio curso e posteriormente como profissional Falkembach (2003). Esta autora também ressalta que o ensino de algoritmos não leva em consideração que cada aluno possui de forma individualizada um potencial intelectual, assim como as habilidades e que isto acarreta diferentes desempenhos na aprendizagem. O sistema tradicional de ensino não considera as diferenças dos alunos, suas aptidões e dificuldades, principalmente em conteúdos em que as habilidades e o raciocínio lógico são extremamente necessários. Assim, é necessário construir estratégias educacionais que possibilitem ao aluno encontrar as possíveis soluções para o problema de forma lógica e coerente.

Durante a prática docente dos autores constatou-se que diversos alunos relatam que o processo de aprendizagem de algoritmo é difícil e ainda apontam várias causas para estas dificuldades, nas quais podemos destacar o elevado nível de abstração envolvido, as metodologias tradicionais de ensino, com a utilização de quadro branco e caneta, a existência de componentes com experiências e pré-requisitos diversificados sobre os conteúdos, traduzindo-se em ritmos de aprendizagem muito diferenciados e a impossibilidade de um acompanhamento individualizado junto ao aluno.

Em face do exposto, salienta-se que o ensino de algoritmos requer novas estratégias educacionais. Este ensino como suporte para a programação utilizou ao longo dos anos diversas abordagens e ferramentas voltadas ao uso principalmente para ambientes de computador de mesa, convencional também utilizando ambientes Webs. O surgimento dos aparelhos ou dispositivos móveis que começam a ser utilizados pelos jovens adultos que constituem o público alvo predominante no contexto da aprendizagem de algoritmos é incipiente no país, embora existam estudos desenvolvidos no exterior investigando o uso educacional amplo e diversificados de tais dispositivos.

As sessões seguintes deste trabalho apresentarão resultados derivados da análise das experiências internacionais e nacionais bem como investigações preliminares com vistas a avaliar as funcionalidades dos dispositivos predominantemente pelos jovens adultos no Brasil e que poderiam apoiar o ensino aprendizagem de algoritmos.

\section{Uso de Mobile Learning na educação}

A Educação a Distância começa a ingressar na chamada terceira onda tecnológica denominada Mobile Learning, caracterizando-se pelo uso de equipamentos portáteis, em especial computadores de mão, num cenário de "computação pervasiva" pautado pela 
mobilidade global do usuário, conectividade ubíqua, independência de dispositivo e ambiente computacional do usuário disponível em qualquer lugar, a qualquer tempo. Isto também é verdade para o contexto presencial, pois o processo de ensino aprendizagem não ocorre apenas enquanto o aluno está em sala de aula.

O levantamento realizado pelo CETIC (2008) mostra na Tabela 1 a situação brasileira em termos de equipamentos de TIC (Tecnologia de Informação e Comunicação).

\begin{tabular}{|l|c|}
\hline \multicolumn{2}{|c|}{ Proporção de domicílios que possuem equipamentos TIC } \\
\hline \multicolumn{1}{|c|}{ Tipo de equipamento } & $\mathbf{\%}$ \\
\hline Televisão & 98 \\
\hline Antena parabólica & 19 \\
\hline TV por assinatura & 7 \\
\hline Rádio & 87 \\
\hline Telefone fixo & 40 \\
\hline Telefone celular móvel2 & 76 \\
\hline Telefone celular com acesso à internet (Base: Possui aparelho celular) & 23 \\
\hline Console de jogo (vídeo game etc.) & 15 \\
\hline Computador de mesa (Desktop, PC) & 27 \\
\hline Computador portátil (laptop, notebook) & 3 \\
\hline Computador de mão (palmtop etc.) & 0 \\
\hline
\end{tabular}

Fonte: CETIC 2008

Tabela 1: Domicílios brasileiros e TIC

Este levantamento aponta também que o local de uso individual da Internet mais freqüente é a própria casa (36\%) com os centros públicos de acesso pago em segundo lugar (35\%). O trabalho (14\%), casa de outra pessoa (9\%), escola (5\%) e centro público gratuito $(1 \%)$ completam a descrição do contexto. $69 \%$ dos entrevistados despendem entre 1 e 10 horas por semana navegando, a maioria está na faixa etária entre 10 e 24 anos e o percentual dos indivíduos entrevistados que utilizam a Internet para a educação é $72 \%$. Assim pode-se perceber que existe um crescente uso da Informática para uso educacional no Brasil embora os números atuais ainda apontem uma pequena parcela da população com acesso a dispositivos móveis como computadores de mão e telefones celulares com acesso Internet e notebook. Mas este é um segmento que está em franco desenvolvimento principalmente pela evolução dos telefones celulares que atualmente já estão sendo usados por quase todos os brasileiros.

Diversas iniciativas começam a promover em instituições de ensino do Brasil, os primeiros indícios da computação móvel, criando as bases iniciais para novos estágios relacionados à computação pervasiva e ubíqua. O próprio ambiente Moodle, que tem sido tão amplamente usado, já oferece suporte para acesso por dispositivos de pequeno porte, tal como relatado por Ribeiro (2009). Nesse sentido, este trabalho busca identificar as estratégias tecnológicas e pedagógicas que este cenário tem condições de ensejar com base em uma análise de experiências relatadas na bibliografia nacional e internacional embora Marcelino, Mihaylov, \& Mendes (2008) destaquem que as solicitações para utilizar dispositivos móveis tem sido oriundas das áreas de matemática, 
química, física, e para diferentes tipos de aprendizagem não sendo usuais no apoio ao ensino e aprendizagem para a área de algoritmo e programação.

\section{Uso de Mobile Learning no ensino aprendizagem de algoritmos}

O termo Mobile Learning é utilizado para denotar ensino através de aparelhos móveis ou dispositivos móveis, possíveis pelos avanços na tecnologia na área da informática. Apresentam-se como interfaces inteligentes para o usuário utilizando tecnologias de comunicações sem fios, tais como Wi-Fi, Bluetooth assim como as tecnologias do tipo GPS (Global Positioning System), GSM (Global System for Mobile Communications), GPRS (General packet radio service), 3G (3rd Generation), disponíveis em celulares, PDAs (personal digital assistant), palmtops, entre outros. Diversos alunos já possuem dispositivos móveis e alguns sistemas educacionais voltados para o ensino de algoritmo estão sendo construídos tendo em vista este ambiente e outros têm sido adaptados para serem utilizados neste tipo de dispositivo, mas precisam levar em conta as peculiaridades dos dispositivos produzidos por diferentes fabricantes. Isso permitiria que os alunos pudessem ter uma utilização mais flexível e otimizada do seu tempo, uma vez que poderiam usar tais tecnologias sob demanda, em qualquer lugar em qualquer momento ampliando a possibilidade de uso educacional de recursos que hoje já estão sendo utilizados para comunicação e lazer pelos jovens adultos no Brasil.

Os dispositivos móveis têm potencial para oferecer diferentes níveis de envolvimento, mas a maioria das aplicações educacionais investigadas envolve um tipo de utilização dos dispositivos móveis voltado a fornecer o mesmo conteúdo e de forma similar ao que é atualmente feito nos computadores de mesa e notebooks convencionais.

O’Malley (2003) já alertava para o fato de que quando estas tecnologias começassem a ser utilizadas, um diferente paradigma no processo de ensino e aprendizagem, deveria ser construído. A aprendizagem que pode acontecer através dos dispositivos móveis varia conforme o tipo específico de tecnologia e parece diferenciar a aprendizagem móvel de outras formas de aprendizagem. A aprendizagem utilizando dispositivos móveis deverá, por conseguinte, ser ampliada passando a considerar a possibilidades de novas alternativas que poderão ocorrer para um aluno que não está em um ponto fixo, em determinada localização, mas sim fora da sala de aula tradicional e utilizando as oportunidades disponibilizadas pelas tecnologias inerentes aos dispositivos móveis. Dias (2008) aponta algumas características desta aprendizagem que utiliza dispositivos móveis: é usado junto aos alunos em todos os lugares, são considerados como companheiros pessoais são de baixo custo (dependendo do modelo) e fáceis de usar e são utilizados constantemente em todas as esferas da vida e em uma variedade de configurações diferentes.

\subsection{Experimentos envolvendo mobile learning}

Uma pesquisa realizada pela empresa Ericsson em 2002, envolveu o uso destes dispositivos com objetivos especificamente educacionais. Naquele contexto, foram usados aparelhos de diversos modelos como Ericsson R520, Ericsson R380 smartphone e outros. Nove alunos com menos de 24 anos participaram do curso e a Tabela 2 mostra 
resultados dos sentimentos e sensações dos alunos no que tange ao uso destes dispositivos.

\begin{tabular}{|c|c|c|c|c|}
\hline Consulta & $\begin{array}{l}\text { Concordaram } \\
\text { positivamente }\end{array}$ & Concordaram & Incerto & $\begin{array}{c}\text { Não } \\
\text { Concordaram }\end{array}$ \\
\hline Facilidade de utilizar o dispositivo móvel. & 6 & 2 & 0 & 1 \\
\hline $\begin{array}{l}\text { Os participantes se matriculariam em outro curso } \\
\text { utilizando dispositivos móveis. }\end{array}$ & 1 & 5 & 2 & 1 \\
\hline $\begin{array}{l}\text { Se o participante recomendaria mobile-learning } \\
\text { como um modo de estudo para amigos ou colegas }\end{array}$ & 0 & 6 & 0 & 3 \\
\hline Se o celular aumenta a qualidade da aprendizagem & 2 & 5 & 2 & 0 \\
\hline $\begin{array}{l}\text { Através de dispositivos móveis, os objetivos da } \\
\text { aprendizagem foram alcançados. }\end{array}$ & 1 & 6 & 2 & 0 \\
\hline $\begin{array}{l}\text { Facilidade de transferir conteúdos através de um } \\
\text { dispositivo móvel }\end{array}$ & 4 & 2 & 1 & 2 \\
\hline A navegação através do celular no curso foi fácil. & 1 & 5 & 1 & 2 \\
\hline $\begin{array}{l}\text { Avaliação e questionamento no celular do curso foi } \\
\text { eficaz. }\end{array}$ & 0 & 6 & 2 & 1 \\
\hline $\begin{array}{l}\text { Se a utilização mobile na aprendizagem aumenta o } \\
\text { acesso à educação e a formação. }\end{array}$ & 1 & 8 & 0 & 0 \\
\hline
\end{tabular}

Tabela 2 - M-Learning (Fonte Ericsson 2002)

Esta pesquisa aponta dificuldades a serem minoradas nos dispositivos móveis quando em uso para fins educacionais. O volume dos conteúdos que podem ser acessados através de mlearning precisa ser otimizado e minimizado. A exibição de conteúdo da tela dos dispositivos precisa utilizar outras soluções que não a simples redução no tamanho das mensagens exibidas.

Um projeto denominado COMTEXT relatado por Santos (2008) utilizou dispositivos móveis do tipo iPAQs HP 4700 Pocket PC para fins de observação, diário, e-mail, chat, fórum, skype, questionários. O desenvolvimento do COMTEXT está fundamentado na concepção epistemológica interacionista-construtivista-sistêmica Junior (2009). A utilização do COMTEXT contribuiu para uma aprendizagem mais significativa, vinculou a aprendizagem às práticas e processos de mediação pedagógica adotadas pelo professor-orientador, oportunizando uma relação dialógica professor-aluno e a reflexão em relação ao trabalho realizado.

\subsection{Um estudo de caso local}

Alguns experimentos recentes, realizados pelos autores deste trabalho, mostram que os dispositivos móveis existentes no Brasil carecem de mecanismos bastantes usuais disponíveis nos computadores convencionais tal como clicar no URL de um conteúdo e comandar a transferência do mesmo para o computador do aluno guardando uma cópia no dispositivo de armazenamento local. Existem programas que permitem transferir arquivos entre dispositivos móveis e computadores convencionais, mas nem todo o formato de arquivos multimídias tem suporte nos dispositivos móveis. Um estudo de caso visando investigar a portabilidade de conteúdos multimídia mostrou que embora 
exista suporte para arquivos do tipo documentos (.doc, .txt e .pdf), planilha (.xls), imagens (.jpg, .png) o suporte a conteúdos desenvolvidos com a tecnologia Flash ainda é praticamente inexistente o que limita a possibilidade de uso de objetos de aprendizagem construídos com aquela tecnologia. Mesmo no caso de vídeos programas conversores de conteúdo precisam ser usados para produzir arquivos que possam ser exibidos nos dispositivos móveis.

No estudo de caso foi investigada a portabilidade de vídeos obtidos do site http://www.youtube.com com aulas e explicações sobre algoritmos. Tais vídeos embora possam ser baixados usando ferramentas tais como VDownloader precisam também ser convertidos para formatos aceitos em cada tipo de dispositivo o que pode variar. A título de exemplo pode-se observar que no caso de computador de mão Palm TX o formato com a melhor taxa de compressão foi o WMV (Windows Midia Video), mas no caso de Smartphone Palm Centro bem como no telefone celular Sony Ericsson W380 o tipo de arquivo de vídeo usado para exibição da aula nestes dispositivos foi o formato .3gp desenvolvido para telefones celulares de terceira geração.

Um dos desafios atuais é o processo de "baixar" o conteúdo para os dispositivos móveis e de tamanhos reduzidos. Na investigação de soluções constatou-se que o programa Hotsync, usado para transferir os arquivos de vídeo para o computador de mão Palm TX via operação de sincronização, funcionou bem, mas no caso dos telefones celulares dispositivos móveis foram encontradas dificuldades e uma primeira solução envolveu copiar os arquivos diretamente para os cartões de memória a fim de testar aspectos de resolução e percepção de conteúdo visual e auditivo.

Cabe observar que a conversão do vídeo para o formato 3gp (3rd generation file format) resultou em uma redução no tamanho da janela de exibição o que dificultou a leitura dos slides que integravam o vídeo. Outros programas de conversão precisam ser investigados para eliminar esta dificuldade. Nos dois casos a qualidade do áudio foi preservada, mas a característica dos dispositivos móveis (baixa potência sonora) implica na necessidade de uso de alto-falante auricular (fones de ouvido) em ambientes que tenham algum ruído ambiente mesmo que reduzido.

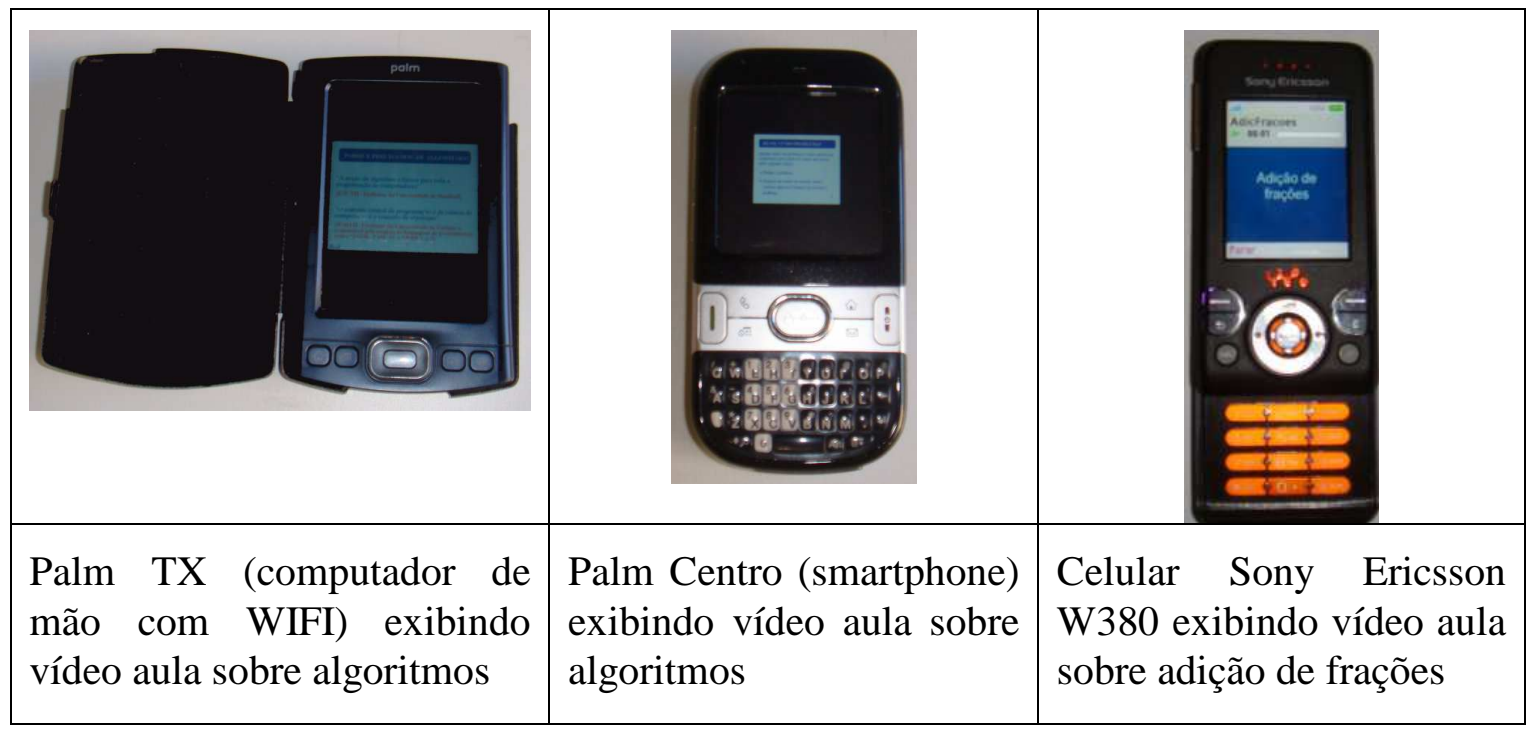

V. $7 \mathrm{~N}^{\mathrm{o}}$ 2, dezembro, 2009 
Figura 2: Vídeos educacionais sobre Algoritmos exibidos em dispositivos móveis típicos

Uma característica diferenciada do computador de mão Palm TX é a possibilidade de desenhar na tela do dispositivo para produzir fluxogramas e depois compartilhar o diagrama com colegas e/ou professor, usando o aplicativo "Anotações", em conjunto com o aplicativo de correio eletrônico "Versa Mail". Com o aplicativo "Anotações" é possível desenhar usando o Stylus (dispositivo de apontar mostrado na figura 4a) sobre a tela sensitiva para produzir uma representação gráfica de um algoritmo (fluxograma).

Após finalizar o fluxograma o próprio programa "Anotações" oferece a opção de enviar a "anotação" usando sistema de Bluetooth, SMS (Short Message Service) ou email. Ao ser escolhida esta última opção, o programa de email (Versa Mail) é ativado no dispositivo e o arquivo com a anotação já aparece como anexo de uma mensagem. Basta então completar os dados do destinatário, o campo com o assunto, algum comentário textual e a mensagem pode ser enviada.

Esta mensagem pode ser exibida em qualquer programa leitor de email e os diagramas criados no computador de mão aparecem em formato de arquivo gráfico que pode ser editado, comentado e encaminhado ou devolvido também como anexo de email e o destinatário pode abrir e acessar o novo desenho tanto em um computador convencional quanto no computador de mão. A Figura 3 ilustra alguns momentos deste processo.

Com este tipo de recurso, fica facilitado o manuseio e a socialização de conhecimento sobre algoritmos, explicitado na forma de fluxogramas. O professor e os colegas podem discutir o diagrama enviado refletindo colaborativamente assincronamente usando email ou acessando um fórum através do navegador, ou mesmo sincronamente, pois existe neste dispositivo um aplicativo que permite interoperar com o popular sistema de chat MSN da Microsoft.
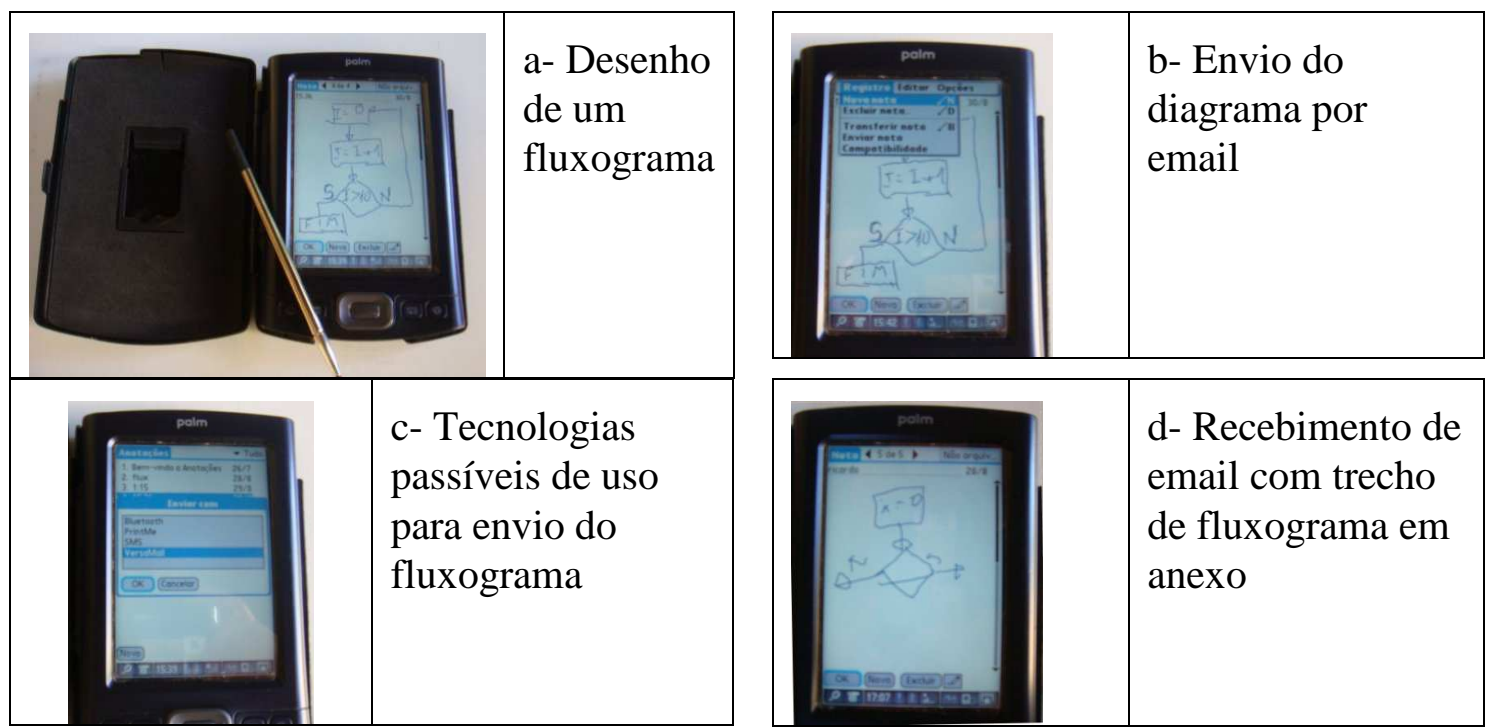

Figura 3: Usando computador de mão para desenhar fluxograma, enviar e receber realimentação. 
Os dispositivos móveis têm potencial para oferecer diferentes níveis de envolvimento e podem ensejar estratégias diferenciadas para oferecer novas oportunidades de interação dos estudantes entre si e com o professor.

A possibilidade de fazer anotações no dispositivo móvel e a socialização das anotações através de alguma sistema de mural eletrônico tem sido apontada como uma vantagem impar deste tipo de equipamento tal como apontado na pesquisa desenvolvida por Fischer (2007).

\section{Conclusões}

Para minimizar as dificuldades e contraponto com as novas metodologias vêm sendo estudadas, principalmente no que diz respeito ao uso de recursos da tecnologia novos artefatos de informática na educação, neste caso estamos propondo a utilização de dispositivos móveis, com o objetivo de potencializar habilidades que visam o desenvolvimento da aprendizagem.

As considerações importantes neste contexto têm como solução encontrar estratégias que contemplem esta aprendizagem, para atender às necessidades dos estudantes, assim como independente da plataforma seja desktop ou dispositivos móveis.

A utilização dos dispositivos móveis no ensino de algoritmos tem a possibilidade de oferecer aos estudantes além da mobilidade e da aprendizagem móvel, diversas outras considerações: i) compreensão dos conceitos de algoritmos envolvendo informação audiovisual; ii) construção de algoritmos usando recursos gráficos (aplicados para desenho de fluxogramas) e envio aos colegas e tutores para socialização e discussão de soluções ampliando as oportunidades de interação; iii) possibilidade de o estudante escolher o módulo a ser baixado para seu equipamento móvel podendo utilizá-lo no local onde tiver disponibilidade e também escolher o estilo de aprendizagem (vídeo, exemplo de fluxogramas, teoria através de textos, animações, exercícios propostos, exercícios propostos pelos estudantes).

A utilização de móbile learning no ensino de algoritmos tem potencial para melhorar o desempenho dos estudantes apoiando colaboração, ensejando o uso de apoio multimídia tal como vídeos, gráficos. Mas cabe ressaltar que as alternativas de comunicação e aplicativos que os dispositivos atualmente no mercado nacional oferecem muitas limitações destacando-se o custo dos dispositivos e da comunicação. Adicionalmente percebeu-se que em função do tamanho da tela e do volume do áudio pode ocorrer interferência de ruído luminoso e sonoro prejudicando a capacidade de focalizar a atenção na atividade educacional. É preciso investigar e testar estratégias para capturar e manter a atenção dos alunos envolvendo-os na atividade de aprendizagem, conforme cita Costabile (2008). Pesquisas na área de cognição e aprendizagem em ambiente em multimídia sendo atualmente desenvolvidas pela equipe buscam subsídios adicionais nesta direção.

\section{Referências bibliográficas}


CETIC. CENTRO DE ESTUDOS SOBRE AS TICS. Pesquisa sobre o uso das Tecnologias da Informação e da Comunicação no Brasil - 2008. Disponível em http://www.cetic.br/ Acessado em Junho de 2009.

Colaborativo. ANAIS DO XXVIII CONGRESSO DA SBC. Belém do Pará, 2008.

COSTABILE, M. F., DE ANGELI, A., LANZILOTTI, R., ARDITO, C., BUONO, P., AND PEDERSON, T. Explore! possibilities and challenges of mobile learning. In Proceeding of the Twenty-Sixth Annual SIGCHI Conference on Human Factors in Computing Systems Florence, Italy, April 05 - 10, 2008. CHI '08. ACM, New York, NY, 1998, p.145-154.

DIAS, A., CARVALHO, J. KEEGAN, D., KISMIHOK, G., MILEVA, N., NIX, J., et al. Introdução ao mobile learning. Ericsson. Mobile learning in action. 2008. Disponível em:http://www.ericsson.com/ericsson/corpinfo/programs/the_role_of_mobile_le arning_in_european_education/products/wp/socrates_wp1_portuguese.pdf, acessado em Ago 2009.

FALKEMBACH, G. A. Uma Experiência de Resolução de Problemas através da Estratégia Ascendente: Ambiente de Aprendizagem Adaptado para Algoritmos. Tese (Doutorado em Informática e Educação), Informática e Educação, Universidade Federal do Rio Grande do Sul, UFRGS, Brasil, 2003.

FISCHER, M. BAIRD, D. Making mlearning work: Utilizing Mobile Technology for active exploration, collaboration, assessment, and reflection in higher education. Journal of Educational Technology systems, 35 (1), 3-30. 2007

GRABE, MARK. GRABE, CINDY. Integrating Technology for Meaningful Learning. Houghton Mifflin Co. Boston. 2001.

JENKINS, T. On the difficulty of learning to program. In Proceedings of 3rd Annual LTSN_ICS Conference Loughborough University, United Kingdom, August 27-29, 2002. The Higher Education Academy, 2002, p.53-58.

JUNIOR, P. G. Aprendizagem com mobilidade (m-learning) nos processos de ensino e de aprendizagem: reflexões e possibilidades, RENOTE, V. 7 nº 1, Julho, 2009. CINTED/UFRGS Porto Alegre-RS, 2009.

MARCELINO, E. R.; ROSATELLI, M. C. Ensino de Programação em um Ambiente

MARCELINO, M.; MIHAYLOV, T.; MENDES, A., "H-SICAS, a handheld algorithm animation and simulation tool to support initial programming learning," Frontiers in Education Conference, 2008. FIE 2008. 38th Annual, Oct. 2008, p.22-25.

MATTOS, M. Construção de abstrações em Lógica de Programação. Anais do XXI Congresso da Sociedade Brasileira de Computação, Fortaleza-CE, 2001.

O'MAlleY, C., VAVOUlA, G., GLEW, J., TAYLOR, J., SHARPLES, M., \& LEFRERE, P. WP 4 - Guidelines For Learning/Teaching/Tutoring In A Mobile, 2003.

PIMENTEL, EDSON. NIZAM, OMAR. Ensino de Algoritmos baseado na Aprendizagem Significativa utilizando o Ambiente de Avaliação NetEdu. WEI/SBC Anais do XXXVIII Congresso da Sociedade Brasileira de Computação, 2008, p.79-88.

RIBEIRO, PATRICK. MEDINA, ROSECLEA. Mobile Learning Engine Moodle (MLE - Moodle): das funcionalidades a validação em curso a distância utilizando 
dispositivos móveis. RENOTE. V.7 N 1, Julho, 2009. CINTED/UFRGS Porto Alegre-RS.

SANTOS, R. P., VIVACQUA, A. S., SOUZA, J. M., \& COSTA, H. A.. Uma Proposta de Cenário para Ensino de Algoritmos e Programação com Contribuições de Cooperação, Colaboração e Coordenação. WEI/SBC Anais do XXXVIII Congresso da Sociedade Brasileira de Computação, 2008.p.157-166 